\title{
A Review: Segmentation Based Objects Analysis in RGB Colored Image using Matlab Simulation
}

\author{
Diksha $^{1}$, Er. Sheetal Chhabra ${ }^{2}$ \\ M. Tech, CSE, KUK, Jagadhri, India ${ }^{1}$ \\ Assistant Professor, CSE Dept., KUK, Jagadhri, India ${ }^{2}$
}

\begin{abstract}
Object Analysis is one of the great challenges of computer vision. The RGB color models are an additive coloration version in wherein red, green and blue light are added collectively in various way to reproduce a large array of colors. The primary goal of the RGB color model is for sensing, representation and show image in electronics systems like televisions and computers, though it's been used in conventional images. There are general phases that define the flaws the working on these Techniques. The proposed work has been explained the concept of the Image Segmentation means to analysis object in RGB colored and retrieve the edge of the image through image segmentation. The analysis of the different objects and quality of image can be implement using image segmentation process.
\end{abstract}

Keywords: Image Processing, Segmentation, Computer Vision

\section{INTRODUCTION}

Image processing is a method to transform an image into digital form and performs some operations on it, so you can get a more advantageous image or to extract some benefit information from it. It is a kind of signal dispensation in which input is image, like video frame or photograph and output can be image or characteristics related to that image. Usually Image Processing system includes treating images as two dimensional signals even as making use of already set signal processing strategies to them.

It is among rapidly developing technologies today, with its applications in various aspects of a business. Image Processing forms core research area inside engineering and computer science disciplines too.

Image processing basically include the following steps:

- Importing the image with optical scanner or by way of digital photography.

- Reading and manipulating the image which incorporates data compression and image enhancement and recognizing style those aren't to human eyes like satellite TV for pc image.

- Output is the remaining stage wherein result may be altered image or report that is based on image analysis.

There are two varieties of methods used for image processing namely, analogue, and digital image processing. Analogue image processing can be used for the hard copy like printouts and photographs. Image analyses use various basic of interpretation at the same time as using these visual techniques. The three general phases that each one types of data through on the equal time as usage of digital technique are pre-processing, enhancement, and show, information extraction. The three general phases that all types of data have to undergo while using digital technique are preprocessing, enhancement, and display, information extraction. Computer vision deals with developing a system in which the input is an image and the output is some information.
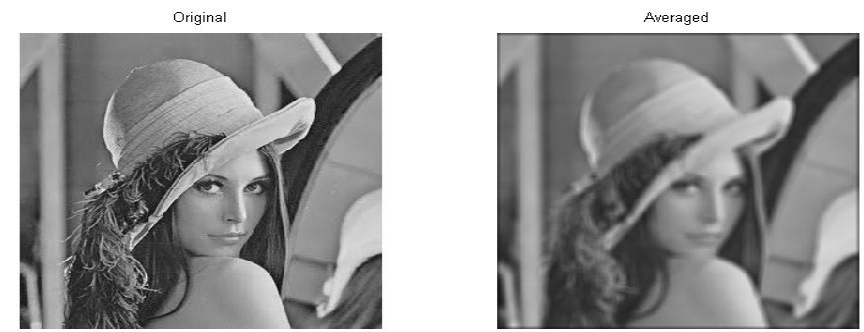

Figure 1: Example of Image Processing

For example: Developing a system that scans human face and opens any kind of lock. This system would look something like this. Importance and necessity of digital image processing steps from two principal application areas: the first being the Improvement of pictorial information for human interpretation and the second being the Processing of a scene data for an autonomous machine perception. Digital image processing has a broad range of applications such 
as remote sensing, image and data storage for transmission in business applications, medical imaging, acoustic imaging, Forensic sciences and industrial automation.

Image segmentation is the department of an image into regions or categories, which correspond to exceptional objects or parts of objects. Every pixel in an image is allocated to one of a number of these categories. A good segmentation is typically one in which:

- $\quad$ Pixels in the equal category have comparable gray scale of multivariate values and form a connected region.

- Neighbouring pixels which might be in exceptional categories have dissimilar values.

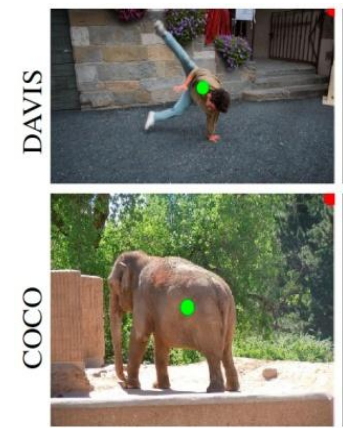

(a) Input
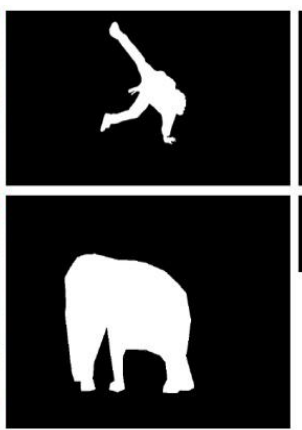

(b) Ground truth
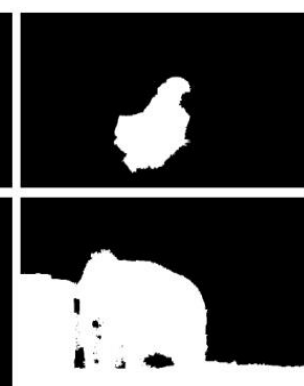

(c) Graph cuts
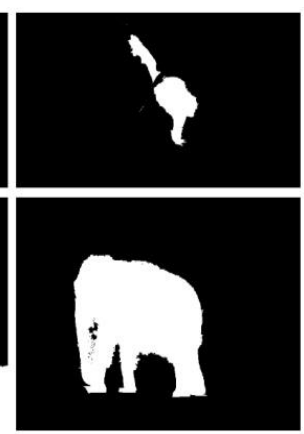

(d) DOS
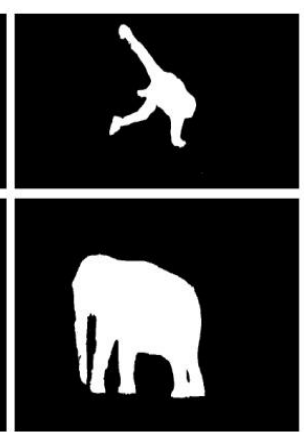

(e) Our approach

Figure 2: Image Segmentation

Image segmentation is initial or front stage processing of image compression. The performance of segmentation process is its speed, correct form matching and higher shape connectivity with its segmenting result. Segmentation refers to the technique of figuring out and keeping apart the surface and regions of the digital image which corresponds to the structural units. Segmentation may depend on various features which are contained within the image. It is able to be both color and texture. The image segmentation problem is concerned with partitioning an image into multiple regions in line with some homogeneity criterion. This article is main concerned with graph theoretic processes to image segmentation. Segmentation-based object categorization can be viewed as a specific case of spectral clustering implemented to image segmentation.

\section{Classification of Image Segmentation:}

In image segmentation process, the basic step is espect detection. It divides an image into object and its background. Edge detection divides the image by observing the change in intensity or pixels of an image. Gray histogram and Gradient are two most important methods for detecting edge detections in image segmentation. Edge detection operators are divided into categories as first order derivative operators and second order derivative operators. Second order operators give reliable results. The canny edge detector is a second derivative operator.
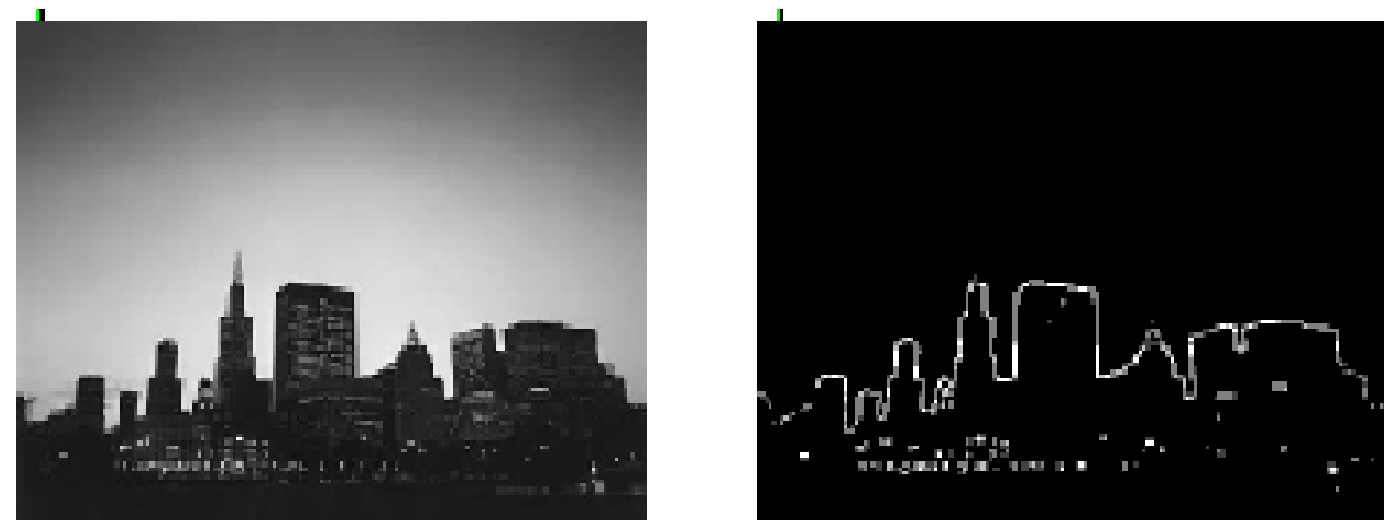

Figure 3: Segmentation by Edge Detection

One of the simplest processes to segment an image is based on the intensity levels and is referred to as threshold based. Thresholding may be applied both globally or locally. Global thresholding distinguishes object and background pixels through comparing with threshold value selected and use binary partition to segment the image. Local thresholding is also known as adaptive thresholding. On this approach the threshold value varies over the image depending on the local characteristic of the subdivided regions in the image. Histogram thresholding is used to segment the given 
image, there is positive pre-processing and post-processing techniques required for threshold segmentation. The Priniciple thresholding techniques proposed by way of unique researchers are Mean method, P-tile method, Histogram dependent technique, Edge Maximization technique, and visual technique.

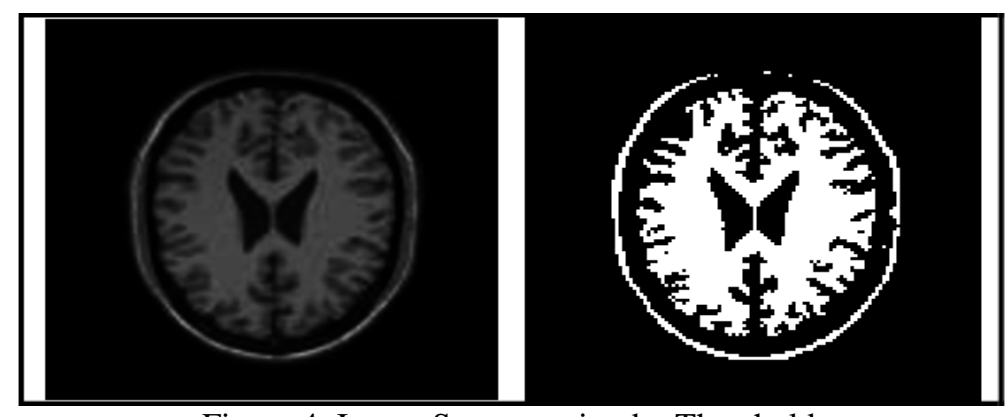

Figure 4: Image Segmentation by Threshold

An example shows that color thresholding can focus on an object of interest much better than its grayscale analogue. The RGB Color space distance thresholds are specified for the R, G, and B components.

\section{Applications}

1. Image Compression: Segment the image into homogeneous components, and use the maximum suitable compression algorithm for every component to improve compression.

2. Medical Diagnosis: Automatic segmentation of MRI images for identify of cancerous regions.

3. Mapping and Measurement: Automatic analysis of remote sensing data from satellites to identify and measure regions of interest.

4. Transportation: Partitions a transportation network makes it feasible to discover regions characteristic by homogenous traffic state.

\section{Benefits}

1. Segmentation accuracy determines the eventual achievement or failure of automated analysis procedure.

2. Development of pictorial information for human perception/interpretation.

3. Mapping and Measurement: Automatic analysis of remote sensing data from satellites to identify and measure regions of interest. E.g. Petroleum reserves.

4. It might be viable to research the image in the computer and provide cues to the radio logistics to assist detect important/suspicious structure.

\section{LITERATURE REVIEW}

In this paper Author's has explain about Region Based Segmentation and Object Detection. Object and multi-class image segmentation are carefully associated responsibility that may be greatly improved while solved collectively by feeding information from one task to the other, but existing state-of-the-art models use a separate representation for each task making joint inference clumsy and leaving the classification of many component of the scene ambiguous. In this work, they recommend a hierarchical region-based approach to joint object detection and image segmentation. Their technique simultaneously reasons about pixels, regions and objects in a coherent probabilistic version. Pixel appearance features allow us to perform well on classifying amorphous background classes, when the express illustration of areas facilitate the computation of extra sophisticated features essential for object detection. Importantly, version offers a single unified description of the scene-they explain each pixel in the image and put in force worldwide consistency among all random variables in model. They run experiments on the challenging Street Scene dataset and show vast improvement over state-of-the-art results for object detection accuracy [1].

On this paper they study the problem of the detection of semantic objects from known categories in images. Unlike existing techniques which perform at the pixel or at a patch stage for recognition, they propose to rely on the categorization of image segments. Previous work has highlighted that image segments offer a sound support for visible object class recognition. In this work, they use image segments as primitives to extract strong features and train detection models for a predefined set of class. Several segmentation algorithms are benchmarked and their performances for segment recognition are as compared. They propose two methods for enhancing the segments classification, one based on the fusion of the classification results obtained with the different segmentations, the other one based on the optimization of the global labelling by way of correcting local ambiguities between neighbour segments. They use as a benchmark the Microsoft MSRC-21 image database and display that approach competes with the current state-of-the-art [2]. 
This paper Author's has investigates segmentation based interest points for matching and recognition. They propose two simple methods for extracting capabilities from the segmentation maps, which focus on the boundaries and centers of the gravity of the segments. In addition, this will taken into be consideration a singular approach for comparing unsupervised image segmentation algorithms. Former evaluations purpose at estimating segmentation quality by using how properly resulting segments adheres to the contours apart ground-reality foregrounds from backgrounds and therefore explicitly focus on particular objects of interest. In comparison, they suggest to measure the robustness of segmentations by the repeatability of features extracted from segments on images related by various geometric and photometric alterations. Similarly, their evaluation presents a new insight into suitability of the segmentation methods for producing nearby features for image retrieval or recognition. Several segmentation methods are evaluated and as compared to state-of-the art interest point detectors the usage of the repeatability criteria in addition general matching and recognition benchmarks.[3]

In this paper Author's has address the problem of object detection and segmentation usage of global holistic properties of object form. Worlwide shapes representations are highly susceptible to clutter inevitably present in realistic images, and may be applied robustly only using a precise segmentation of the object. To this end, they propose a figure/ground segmentation approach for extraction of image regions that resemble the worldwide properties of a model boundary structure and are perceptually salient. Their shape representation, known as chordiogram, is based on geometric relationships of object boundary edges, even the perceptual saliency cues they use favour coherent regions distinct from the background. They formulate the segmentation problem as an integer quadratic program and use a semi specific programming relaxation to resolve it. Obtained solutions provide the segmentation of an object as well as a detection score used for object recognition. Their single-step method achieves state-of-the-art performance on numerous object detection and segmentation benchmarks. [4]

In computer vision extracting an object from an image automatically is too hard. Towards addressing this issue a complete evaluation of most of the Object detection through one of the kind Segmentations is executed taken from the major current publications covering various aspects of the research in this area. They identify the following methods of the state-of-the-art techniques in which an object can be detected:

(1) Mean Shift Segmentation with Region Merging,

(2) Boundary Structure Segmentation with Region Grouping,

(3) Watershed Segmentation with Region Merging.

All these are semi automatic detection of an object via segmentation and contour based shape descriptor. The results tabulated show that the Mean Shift Segmentation with Region Merging Technique yields the pleasant end result over the opposite two methods in detection the Object of Interest. [5].

\section{OBJECTIVES}

The aim of this thesis is to develop segmentation based object analysis for RGB Colored imaging applications. There are many ways to overcome the problem during image segmentation. In previous research paper, authors have addressed the problem of object detection and segmentation using global holistic properties of object shape. One of the difficulties in previous model is learning the trade-off between energy terms - too strong a boundary penalty and all regions will be merged together and many problem addresses during optimization. We formulated the evaluation of image segmentation methods for an optimization problem and formulate the problem of curve/surface etc. To fulfil the problem and maintain the accuracy, we need to follow the following objectives:

1 To study and analysed the Segmentation based Object Analysis.

2. To identify the quality of image by image segmentation.

3. To retrieve the edges of the image by image segmentation.

4. Implement proposed work in MATLAB and evaluate results.

\section{PROPOSED METHODOLOGY}

1. Analyse the Existing Phenomena's of the pre-defined techniques

2. Study the Techniques and working of the segmentation

3. Define the flaws in the working and research on these Techniques for identification.

4. Identify the problems in existing techniques and Methods.

5. Flow Development of new proposed technique

6. Develop the Algorithm in Simulation Tool.

7. Implementation in MATLAB for generate the results. 
Vol. 8, Issue 5, May 2019

\section{CONCLUSION AND FUTURE WORK}

In this research topic we described the Segmentation Based Objects Analysis in RGB colored image. Our approach to analyse and identify the quality of object and image by image segmentation and retrieve the edges of the object and image by image segmentation in RGB colored.

More work can be possible with analyze multi-scene analysis in RGB colored image and also further research is possible on Segmentation based Object analysis in HEX colored Image or other research like CMYK and PMS colored image.

\section{REFERENCES}

[1]. Stephen Gould, Tianshi Gao and Daphne Koller. "Region Based Segmentation and Object Detection".

[2]. Remi Vieux, Jenny Benois-Pineau, Jean-Philippe Domenge and Achille Braquelaire."Segmentation-Based multi class semantic objects Detection" 2011.

[3]. Piotr Koniusz and Krystian Mikolajczyk "Segmentation Based Interest Points and Evaluation of Unsupervised Image Segmentation Methods", CVSSP, University of Surrey, UK

[4]. Alexander Toshev, Ben Taskar, Kostas Daniilidis "Shape-based Object Detection via Boundary Structure Segmentation" 2011.

[5]. Pushpalatha S. Nikkam ; Nagaratna P. Hegde ; Eswar Reddy, "Comprehensive analysis of object Detection through Segmentation", 2014 Fifth International Conference on Signal and Image Processing. 